\title{
Improving Effect of Promoting Cerebral Circulation on Patients with Senile Dementia
}

\author{
Mingmin Deng \\ Tongji Medical College of Huazhong University of Science and Technology, Wuhan 430030, Hubei, China \\ Email: dengmingmin@hust.edu.cn
}

\begin{abstract}
Purpose - To explore the improving effect of promoting cerebral circulation on patients with senile dementia. Methods - 96 patients with senile dementia admitted from January 2018 to December 2019 were selected and divided into A (conventional treatment) and B (conventional + promoting cerebral circulation treatment) groups, 48 cases in each group, according to the computerized flow grouping method. The clinical therapeutic effects of the two groups were compared and analyzed. Results - (1) Compared with Group A, the total effective rate of Group B was higher $(95.83 \%>83.33 \%)$ $\left(x^{2}=4.019, P=0.045\right)$; (2) Before treatment, there was no significant difference between groups $(P>0.05)$. After treatment, compared with Group A, Group B had higher MMSE score, lower ADAS-cog score, HAMD score and ADL score $(P<0.05)$; (3) During the clinical treatment of the two groups, no obvious adverse reactions were seen. Conclusion - The treatment of promoting cerebral circulation in patients with senile dementia can not only effectively improve the symptoms of dementia, but also improve the ability of daily living. This therapy is safe and reliable, and is worthy of clinical promotion and use.
\end{abstract}

Keywords: senile dementia, promoting cerebral circulation, improving effect

Dementia is a disease with a high incidence in the elderly, and it is a kind of central nervous system disease. Its clinical manifestations are memory loss, cognitive impairment and so on. While it brings pain to the physical and mental health of patients, it also brings harm to their families ${ }^{[1]}$. If it is not treated in time, the condition will aggravate; the episodes will be frequent; the patient's depression and restlessness will aggravate; the condition will deteriorate faster ${ }^{[2]}$. Therefore, the clinical research of senile dementia is very important. Based on this, this study aims to explore the improving effect of promoting cerebral circulation by giving patients with senile dementia promoting cerebral circulation. The description is as follows.

\section{Materials and methods}

\subsection{General materials}

The research period is from January 2018 to December 2019. According to the computerized flow grouping method, 96 patients with senile dementia were divided into 2 groups, 48 cases in each group. In Group A, gender: male/ female=26/22; age: 78 years old (oldest), 60 years old (youngest), mean $(72.38 \pm 2.13$ ) years; disease course: 8 years (longest), 1 year (shortest), mean (4.53 \pm 1.21$)$ years; complications: 20 cases of hyperlipidemia, 16 cases of hypertension, 12 cases of atherosclerosis. In Group B, gender: male/female=28/20; age: 80 years old (oldest), 60 years old (youngest), mean (72.69 \pm 2.09$)$ years old; disease course: 9 years (longest), 1 year (shortest), mean $(4.42 \pm 1.32$ ) years; complications: 20 cases of hyperlipidemia, 14 cases of hypertension, 14 cases of atherosclerosis. The clinical data of the two groups are comparable $(P>0.05)$.

Enrollment criteria: (1) the selected patients were all patients with senile dementia, who were diagnosed by EEG and neuroimaging examination; (2) the selected patients had obvious memory deterioration, slow movement, and sluggish eyesight; (3) more than 60 years old; (4) the research is in line with medical ethics; (5) instruct patients to sign an informed letter, and patients and their families have the right to know. Elimination criteria: (1) those with severe respiratory disease; (2) those with liver function and immune dysfunction; (3) those with tumor disease; (4) those with severe unstable cardiovascular disease; (5) cerebral infarction/brain atrophy; (6) those who cannot cooperate with the researcher.

\subsection{Methods}

Patients in Group A were given conventional treatment, that is, symptomatic treatments such as improving cerebral blood supply, maintaining blood sugar and blood pressure stability, and anti-platelet aggregation.

Patients in Group B received conventional + promoting cerebral circulation treatment, and the conventional treatment 
was the same as that in group A. Promoting cerebral circulation: according to the principle of promoting cerebral circulation recovery, give Oxiracetam capsules (Harbin Sanlian Pharmaceutical, H20184000), 0.4g/time, 2 times/d, 1 course of treatment $=1$ month.

Both groups of patients were treated continuously for 3 months. During the treatment, the two groups of patients were prohibited from anticholinergic drugs and memory enhancers.

\subsection{Observation indicators}

The clinical efficacy, MMSE score, ADAS-cog, HAMD score, and ADL score were used as evaluation scales to evaluate the improving effect of promoting cerebral circulation treatment on patients with senile dementia.

The clinical curative effect is judged, taking the patient's dementia symptoms, mental recovery, and self-care ability as evaluation indicators, and the clinical curative effect is divided into markedly effective (significantly improved), effective (improved), and ineffective (no change or even worse). Markedly effective + effective $=$ total effective rate.

Relevant scale score: MMSE (mental state), ADAS-cog (cognitive function), HAMD (depressive mood), ADL (daily life). Among them, MMSE: total score 30 points, high score, good mental state; ADAS-cog: total score 75 points, high score, poor cognitive function; HAMD: high score, severe depression; ADL: total score 56 points, high score, poor selfcare ability.

Safety evaluation, recording the occurrence of adverse reactions during the treatment of the two groups of patients.

\subsection{Statistical processing}

The statistical software SPSS25.0 was used; qualitative data such as the total effective rate of treatment (\%) and measurement data such as related scales $(\overline{\mathrm{x}} \pm \mathrm{s})$ were subjected to $x^{2}$ test and $t$ test respectively; $P<0.05$, indicating that the data are different.

\section{Results}

\subsection{Comparison of clinical efficacy between the two groups of patients}

Compared with Group A, the total effective rate of Group B is higher $(95.83 \%>83.33 \%)\left(x^{2}=4.019, P=0.045\right)$, as is shown in Table 1.

Table 1. Comparison of clinical efficacy between the two groups of patients [n(\%)]

\begin{tabular}{cccccc}
\hline Group & Number of cases & Markedly effective & Effective & Ineffective & Total effective rate \\
\hline Group A & 48 & $25(52.08)$ & $15(31.25)$ & $8(16.67)$ & $83.33(40 / 48)$ \\
Group B & 48 & $33(68.75)$ & $13(27.08)$ & $2(4.17)$ & $95.83(46 / 48)$ \\
$x^{2}$ & - & - & - & - & - \\
\\
$P$ & - & - & - & 0.045 & - \\
\hline
\end{tabular}

\subsection{Comparison of relevant scales between the two groups of patients}

Before treatment, there was no significant difference between the groups $(P>0.05)$. After treatment, compared with group A, group B's MMSE score was higher, and ADAS-cog score, HAMD score, and ADL score were lower $(P<0.05)$. See Table 2 .

Table 2. Comparison of related scales between the two groups of patients ( \pm s, points)

\begin{tabular}{|c|c|c|c|c|c|}
\hline Related scale & Time & Group A $(n=48)$ & Group B $(n=48)$ & $t$ & $P$ \\
\hline \multirow{2}{*}{ MMSE score } & Before treatment & $12.13 \pm 1.49$ & $12.17 \pm 1.52$ & 0.130 & 0.897 \\
\hline & After treatment & $16.52 \pm 1.34^{*}$ & $22.83 \pm 2.24^{*}$ & 16.748 & 0.000 \\
\hline \multirow{2}{*}{ ADAS-cog score } & Before treatment & $34.58 \pm 2.81$ & $34.67 \pm 2.79$ & 0.157 & 0.875 \\
\hline & After treatment & $27.34 \pm 2.28^{*}$ & $21.36 \pm 2.35^{*}$ & 12.653 & 0.000 \\
\hline \multirow{2}{*}{ HAMD score } & Before treatment & $15.73 \pm 2.13$ & $15.68 \pm 2.17$ & 0.114 & 0.910 \\
\hline & After treatment & $11.85 \pm 1.97^{*}$ & $7.23 \pm 1.48^{*}$ & 12.990 & 0.000 \\
\hline \multirow{2}{*}{ ADL score } & Before treatment & $35.68 \pm 5.26$ & $35.49 \pm 5.19$ & 0.178 & 0.859 \\
\hline & After treatment & $23.39 \pm 1.39^{*}$ & $15.68 \pm 5.23^{*}$ & 9.871 & 0.000 \\
\hline
\end{tabular}


Note: Compared with before treatment, ${ }^{*} P<0.05$.

\subsection{Safety evaluation of the two groups of patients}

During the period of clinical treatment, there were no obvious adverse reactions in the two groups.

\section{Discussion}

Senile dementia is a neurological disease with a high incidence, which has the characteristics of concealed onset, slow progress, and high disability or fatality rate. The main pathogenesis of the disease has not been found clinically, but many studies have shown that the occurrence of the disease is related to factors such as lower acetylcholine levels and focal cerebral ischemia ${ }^{[3]}$. According to reports ${ }^{[4]}$, the prevalence of senile dementia in China has been increasing in recent years. If it is not treated in time, it is very easy to cause damage to the patients' nerve function, cause physical, language, and cognitive dysfunction, and reduce the quality of life of the patients. However, there is no curative drug for the treatment of senile dementia, so the clinic is mostly based on the clinical manifestations and pathological changes of patients, using neuroprotection, cholinesterase inhibitors and other symptomatic treatments ${ }^{[5]}$.

Based on the above-mentioned situation, this study has taken the treatment of promoting cerebral circulation in patients with senile dementia, and achieved good results. Promoting cerebral circulation therapy can improve clinical efficacy and improve the quality of life of patients. In the promoting cerebral circulation, the patient not only received symptomatic treatment, but also received oxiracetam drug treatment. Oxiracetam is a derivative of 3-hydroxy-4aminobutyric acid, which is a kind of nootropic drug. It can stimulate adenylate kinase, enhance the transport capacity of acetylcholine (cerebral cortex, hippocampus), increase the uptake of glucose and oxygen by brain cells, improve patients' memory impairment, and activate neurons in the system. The production of acetylcholine increases the amount of protein synthesis, thereby increasing the patient's memory ${ }^{[6]}$. As a drug that can improve intelligence, Oxiracetam can promote the organic combination of phospholipid ethanolamine and phospholipid choline, strengthen the structure of cell membranes (neurons), and increase structural stability. In addition, when it exerts its effects, Oxiracetam can ignore the blood-brain barrier and help restore normal central nervous pathways, thereby fully increasing the utilization of neurons and increasing the energy metabolism of the patient's brain, and restoring damaged neurons and improving. The function of brain tissue has a real effect on the recovery of patients' memory and learning ability ${ }^{[7]}$.

This study used MMSE, ADL and other scales to explore the effect of promoting cerebral circulation on patients with senile dementia. Among them, MMSE is a commonly used cognitive examination scale for senile dementia, through which patients with suspected senile dementia can be detected in time for timely treatment. The ADL is an assessment scale for self-care ability in daily life, which is suitable for judging the independent social activity ability of subjects. However, affected by the disease, it is more difficult for patients with dementia to complete simple actions. Therefore, the ability to take care of daily life of the patients with dementia is judged before treatment, which helps to understand the patient's condition. In this study, patients in group A received conventional treatment, and patients in Group B received conventional + cerebral circulation promotion treatment. Finally, it is concluded that the total effective rate of Group B is higher than that of Group A $(95.83 \%>83.33 \%)\left(x^{2}=4.019, P=0.045\right)$. It can be seen that, compared with conventional treatment, the implementation of treatment to promote cerebral circulation is beneficial to the improvement of the patient's condition and can significantly improve the patient's dementia. At the same time, after treatment, the MMSE score of group B was higher than that of group A, and the ADAS-cog score, HAMD score and ADL score were lower than those of group A $(P<0.05)$. Judging from the MMSE, ADAS-cog, ADL and other scales, the promotion of cerebral circulation therapy is effective for patients with dementia. In addition, the two groups of patients had no obvious adverse reactions during the treatment period, which proved that the treatment of promoting cerebral circulation is reliable and safe. The reason is that the promotion of cerebral circulation treatment is to use oxiracetam drugs to improve the blood volume of the ischemic area, thereby increasing its oxygen supply, helping to promote the metabolism of glucose and oxygen in nerve cells, thereby improving nerve cells Hypoxia tolerance and change the function of mitochondria, thereby improving the condition of senile dementia.

In summary, using the promotion of cerebral circulation in patients with senile dementia can not only significantly improve the patient's condition, but also improve the patient's depression, improve the patient's daily self-care ability, and improve the patient's quality of life. It is worth applying and recommending. 


\section{References}

[1] Wang Weifeng, Zhang Huitian, Shi Dehai. Observation on the effect of comprehensive rehabilitation exercise combined with drugs in the treatment of senile dementia. Chinese Convalescent Medicine. 2020; 29(10): 1083-1084.

[2] Liu Jiabei, Li Jieru. The influence of individualized music nursing intervention on the behavior of patients with dementia. Shanxi Medical Journal. 2020; 49(16): 2222-2224.

[3] Wang Jia. Observation of the clinical effect of donepezil hydrochloride treatment in patients with senile dementia. China Modern Medicine Application. 2020; 14(18): 205-207.

[4] Wang Jindong, Zhou Tiantian, Chen Xiaoqin, et al. Clinical effect of magnesium valproate combined with donepezil in the treatment of patients with senile dementia. Chinese Medicine. 2020; 15(5): 699-703.

[5] Lu Jiyang. Study on the clinical effect and safety of donepezil in the treatment of senile dementia. Chinese Medical Guide. 2020; 18(18): 134-135.

[6] Zhang Xinyan. Observation of the clinical effect of amisulpride combined with oxiracetam on cognitive and mental disorders in patients with senile dementia. China Health Care and Nutrition. 2020; 30(20): 155.

[7] Geng Xueyu. The clinical efficacy of oxiracetam combined with donepezil hydrochloride in the treatment of patients with senile dementia. World Latest Medicine Information. 2019; 19(40): 129-130. 